\title{
Origin of Light-Induced Photophysical Effects in Organic Metal Halide Perovskites in the Presence of Oxygen
}

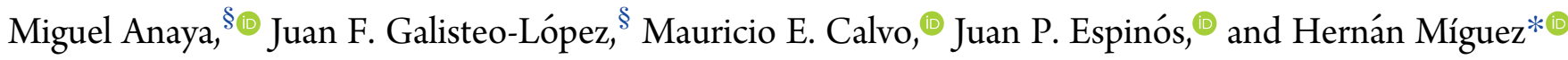 \\ Instituto de Ciencia de Materiales de Sevilla, Consejo Superior de Investigaciones Científicas-Universidad de Sevilla, C/Américo \\ Vespucio 49, 41092 Sevilla, Spain
}

\section{Supporting Information}

ABSTRACT: Herein we present a combined study of the evolution of both the photoluminescence (PL) and the surface chemical structure of organic metal halide perovskites as the environmental oxygen pressure rises from ultrahigh vacuum up to a few thousandths of an atmosphere. Analyzing the changes occurring at the semiconductor surface upon photoexcitation under a controlled oxygen atmosphere in an X-ray photoelectron spectroscopy (XPS) chamber, we can rationalize the rich variety of photophysical phenomena observed and provide a plausible explanation for light-induced ion migration, one of the most conspicuous and debated concomitant effects detected during photoexcitation. We find direct evidence of the formation of a superficial layer of negatively charged oxygen species capable of repelling the halide anions away from the surface and toward the bulk. The reported PL transient dynamics, the partial recovery of the initial state when photoexcitation stops, and the eventual degradation after intense exposure times can thus be rationalized.

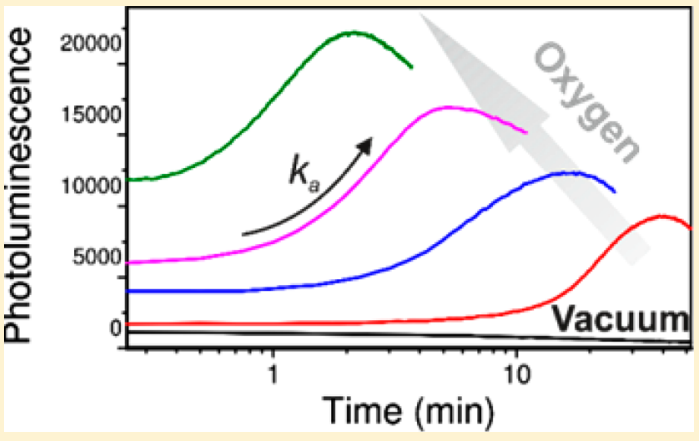

Thaser. he irruption of hybrid organic-inorganic perovskite devices $^{1-5}$ in the field of optoelectronics has generated great interest in the analysis of the fundamental processes that lie behind their optical and electrical properties. ${ }^{6-9}$ These materials are generically formulated as $\mathrm{ABX}_{3}$, where $\mathrm{A}$ is an organic cation $\left(\mathrm{CH}_{3} \mathrm{NH}_{3}{ }^{+}, \mathrm{CH}\left(\mathrm{NH}_{2}\right)_{2}^{+}\right), \mathrm{B}$ a metal cation $\left(\mathrm{Pb}_{2}{ }^{+}, \mathrm{Sn}_{2}{ }^{+}\right)$, and $\mathrm{X}$ a halide anion $\left(\mathrm{I}^{-}, \mathrm{Br}^{-}, \mathrm{Cl}^{-}\right)$. While they present large optical absorption, efficient photocarrier transport, and high photoemission or electroluminescence quantum yield, the instability of these properties is also one of their most distinctive features. ${ }^{10}$ One of the main sources of instability is that induced by external irradiation, which has been further established to depend strongly on the environment. ${ }^{11-15}$ In particular, analysis of the temporal evolution of the photoemission is a means to readily identify and characterize the magnitude of the changes observed. In brief, simultaneous exposure to gaseous oxygen and light causes an initial increase of several orders of magnitude of the luminescence (photoactivation). Upon continuous excitation, luminescence will eventually start dropping and reach values lower than the initial ones, an effect that is accompanied by decomposition of the semiconductor (photodeactivation). The dynamics, magnitude, and reversibility of this latter process depend strongly on illumination intensity and exposure time. Remarkably, all of these modifications of the luminescence are accompanied by a redistribution of the ions, which move away from the illuminated areas ${ }^{16,17}$ and whose driving force is currently being debated.

Herein we show a systematic study of the effect that the simultaneous occurrence of photoexcitation and oxygen in the environment has on both the photophysical properties and the chemical structure of organic metal halide perovskite films. One of our most interesting observations is that, in order to observe significant photoemission in organic metal halide perovskites, the presence of oxygen in the environment is required, the material being practically inactive before being exposed to this gas. The time evolution of the photoluminescence (PL) with time shows, in turn, a strong dependence on the level of oxygen pressure in the chamber. All of our observations are rationalized based on either wellestablished facts in the field or the results of our own analysis of the modification of the chemical structure of the semiconductor surface by means of XPS. In this regard, we provide evidence that simultaneous exposure of $\mathrm{CH}_{3} \mathrm{NH}_{3} \mathrm{PbX}_{3}$ (in brief, $\mathrm{MAPX}_{3}$ ) perovskite films to oxygen and light with energy above the electronic bandgap yields the formation of anionic oxygen species that accumulate as a negatively charged layer on the semiconductor surface. These reactive species may provide the electrostatic driving force necessary to induce ion migration and defect annihilation, which cause momentary reconstruction of the imperfect bulk lattice and, simultaneously, initiate slow and gradual degradation of the lattice. The mechanism herein proposed provides a satisfactory explanation to, up to now, seemingly contradictory effects, such as the initial enhancement of the photoemission and the subsequent degradation of the organic metal halide perovskites.

Received: June 12, 2018

Accepted: June 21, 2018

Published: June 21, 2018 

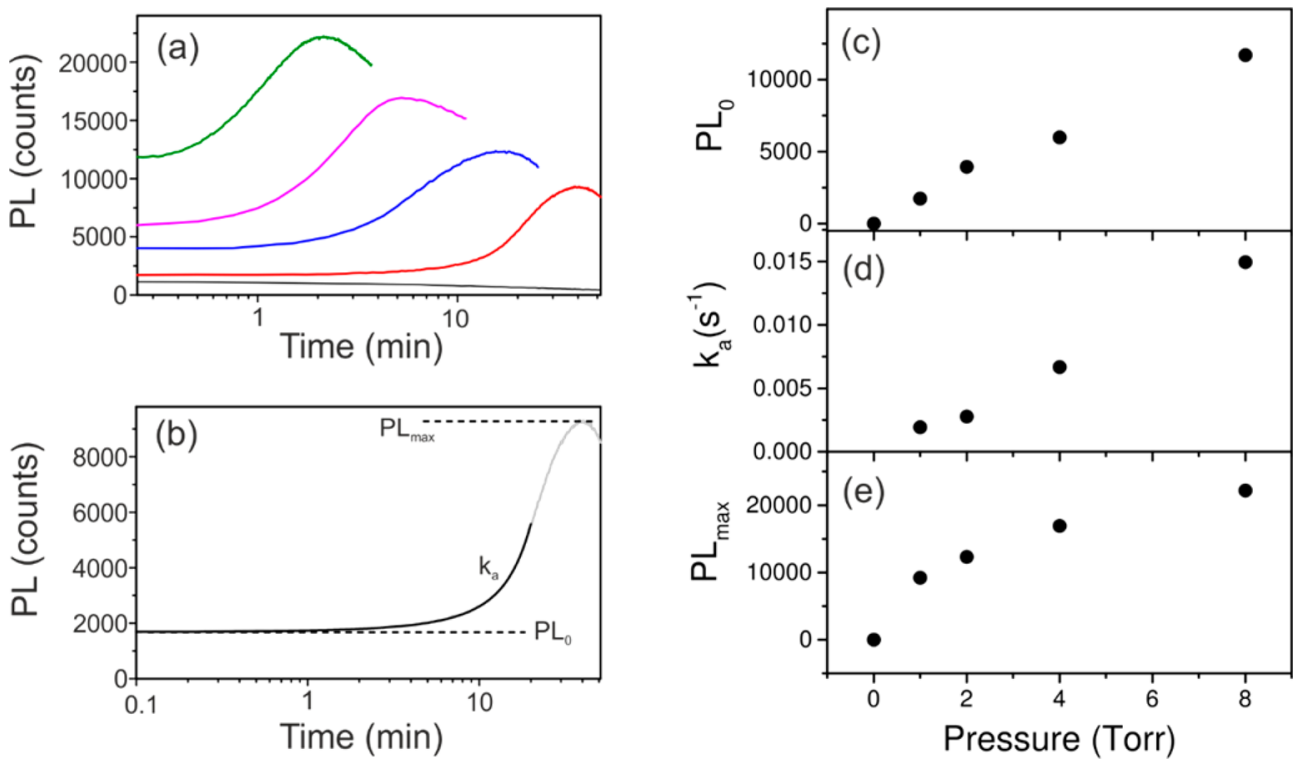

Figure 1. (a) Time evolution of the PL from a MAPBr film exposed to different $\mathrm{O}_{2}$ pressures: $0.75 \times 10^{-8}$ (black), 1 (red), 2 (blue), 4 (magenta), and 8 Torr (green curve). (b) Characteristic PL activation curve (gray line) indicating the initial $\left(\mathrm{PL}_{0}\right)$ and maximum $\mathrm{PL}$ (PL $\mathrm{PLx}_{\mathrm{max}}$ ) and the PL activation constant $\left(k_{\mathrm{a}}\right)$. The black curve represents exponential growth fit. The evolution of these parameters with $\mathrm{O}_{2}$ pressure are shown in $(c-e)$ for $k_{a}, \mathrm{PL}_{\max }$ and $\mathrm{PL}_{0}$.

First, we perform systematic analysis of the dependence of the photophysical properties of an organic metal halide perovskite versus the level of oxygen in the environment. In order to do so, a freshly prepared thin film of $\mathrm{CH}_{3} \mathrm{NH}_{3} \mathrm{PbBr}_{3}$ $(\mathrm{MAPBr})$, synthesized within a glovebox under a controlled oxygen-free $(<0.5 \mathrm{ppm})$ atmosphere (see the Experimental Methods section), is transferred to an ultrahigh vacuum $\left(10^{-8}\right.$ Torr) chamber. Figure 1a displays the PL maximum intensity $(\lambda=533 \mathrm{~nm})$ transients attained under continuous illumination $(\lambda=450 \mathrm{~nm})$ of a MAPBr film both at ultrahigh vacuum (black solid line) and at increasing levels of oxygen in the chamber, namely, 1, 2, 4, and 8 Torr (red, blue, magenta, and green solid lines, respectively). In these latter cases, the phases in which photoactivation and photodegradation are dominant can be readily identified, just as in other hybrid perovskites. ${ }^{11-13}$ However, measurements taken in the absence of oxygen show a slow decay of the PL and no activation whatsoever. This behavior can be understood in light of recent studies realized by Maier and co-workers, ${ }^{18}$ which have demonstrated that the photogeneration of carriers, in the absence of oxygen, induces a strong increase of the density of both halide vacancies in the crystalline structure and neutral iodine in the interstitial sites as a result of enhanced ion conductivity within the material, thus leading to a more defective lattice. This should yield poorer photoemission, as pointed out before by Petrozza et al. ${ }^{14}$ and in good agreement with our observations. In Figure $1 b$, the different parameters analyzed to study the relation between the modifications of the PL observed and the level of oxygen pressure are identified, i.e., background and maximum $\mathrm{PL}$, labeled as $\mathrm{PL}_{0}$ and $\mathrm{PL}_{\max }$ and the rate at which photoactivation occurs, $k_{\mathrm{a}}$. Although all three rise with oxygen pressure in the chamber, the reasons why each one of them increases can be attributed to different processes taking place in the material. The gradual increase of $\mathrm{PL}_{0}$ displayed in Figure 1c implies that the presence of molecular oxygen itself, before photoexcitation starts, is already capable of increasing the PL by orders of magnitude. This observation is in good agreement with the mechanism proposed by De
Angelis, Mosconi, et al. for MAPI, ${ }^{19}$ by which molecular oxygen is capable of temporarily deactivating deep hole traps associated with interstitial iodine, forming relatively stable oxidized species. It can also be observed in Figure 1d that the time required to start observing the PL rise is relatively long (from around 1 to $10 \mathrm{~min}$ for the range of pressures employed) and shortens (i.e., $k_{\mathrm{a}}$ increases) as the oxygen pressure in the chamber is set at a higher value. $\mathrm{PL}_{\max }$ (Figure 1e) shows asymptotic behavior, which indicates that trap passivation may be reaching saturation even for a relatively low oxygen pressure (8 Torr) and short photoexcitation times.

Our observations demonstrate that the dynamics of the photoinduced processes leading to reduced nonradiative carrier recombination and thus higher PL in $\mathrm{MAPX}_{3}$ perovskites strongly depend on the level of molecular oxygen in the environment. While the mechanism described in ref 19 accounts for the increase of background $\mathrm{PL}\left(\mathrm{PL}_{0}\right)$ herein reported, which occurs in the absence of photoexcitation, subsequent light-induced PL activation and deactivation requires a more complex explanation as it has been repeatedly proven to be associated with ion migration. ${ }^{16}$ More specifically, correlated optical microscopy and elemental analysis performed in single crystals demonstrate that illuminated areas become gradually depleted of halide anions, regardless of the perovskite composition and morphology. ${ }^{17,20}$ This migration is partially reversed if the material is left to rest in the dark, but if illumination is maintained for long times or is too intense, visible degradation occurs and PL vanishes irreversibly. Therefore, effects induced by the combination of illumination and oxygen are always accompanied by halide ion migration, which is thought to occur through halogen vacancies. ${ }^{21}$ These facts pose a question about the driving force responsible for ion motion in organic metal halide perovskites when they are photoexcited in the presence of oxygen. Any proposed mechanism of PL enhancement and decay must account for these conspicuous related phenomena.

In order to provide some further insight into this question, we study the changes induced in the chemical structure of the 
inorganic metal halide perovskite surface as a result of the exposure to light with energy above the electronic bandgap (labeled as phase 0), the simultaneous exposure to such light and oxygen (phase I), and while resting in the dark after the treatment (phase II). In order to do so, MAPBr films are introduced in a prechamber of an XPS apparatus, in which ultrahigh vacuum was made. There, they can be photoexcited with blue light $(\lambda=450 \mathrm{~nm})$ and exposed to a controlled oxygen pressure ( 1 Torr), chosen in view of the results shown in Figure 1. The effect of these treatments on the PL is again monitored by measuring in situ the photoemission spectrum through a quartz window, but in this case, modifications of the surface are periodically studied by XPS. For this, ultrahigh vacuum is recovered in the prechamber after oxygen exposure and films are transferred to the main chamber, where they are analyzed without having been exposed to the ambient. The duration of each XPS measurement is indicated using gray shaded backgrounds in Figure 2, where it can be seen that,

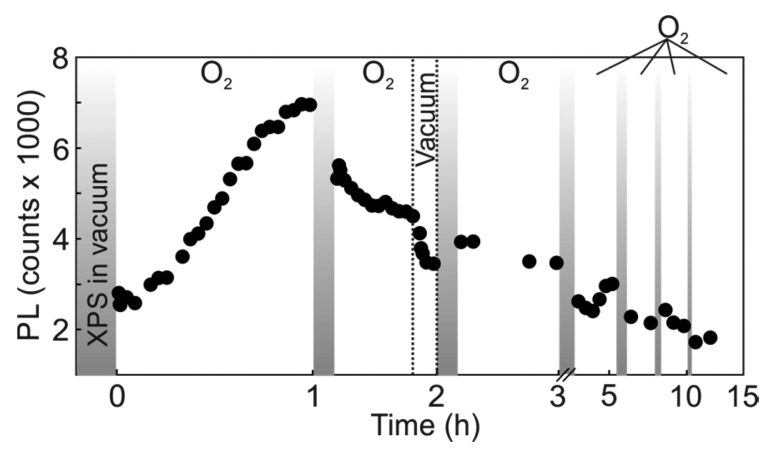

Figure 2. Evolution of the PL maximum intensity $(\lambda=533 \mathrm{~nm})$ of a MAPBr film with time under excitation with monochromatic light of $\lambda$ $=450 \mathrm{~nm}$ in a 1 Torr $\mathrm{O}_{2}$ atmosphere. The time required to perform XPS acquisition at ultrahigh vacuum is also indicated (gray regions).

overall, the PL intensity follows a similar trend as the one observed in the transient displayed in Figure 1a. Full details on the experimental setup, as well as on initial tests without light and oxygen, can be found in the Experimental Methods section and in the Supporting Information (Figures S1 and S2).

The evolution of the amount of all relevant elements, namely, lead, bromine, nitrogen, and carbon, was monitored through the changes in the $\mathrm{Pb} 4 \mathrm{f}, \mathrm{Br} 3 \mathrm{~d}, \mathrm{~N}$ 1s, and $\mathrm{C} 1 \mathrm{~s}$ XPS signals. As the amount of $\mathrm{Pb}$ at the surface remained almost constant during the whole analysis, as revealed by the integral of the corresponding signal, we use it as a reference to estimate the changes in the concentration of the rest of the elements. First, the XPS analysis of the MAPBr during phase 0 (vacuum and light) showed that photoexcitation gives rise to a sequential transformation of $\mathrm{Pb}^{2+}$ into $\mathrm{Pb}^{0}$, while the signals of bromide, nitrogen, and carbon remain stable (see Figures S4 and S5). Degradation of the $\mathrm{CH}_{3} \mathrm{NH}_{3} \mathrm{PbI}_{3}$ (MAPI) perovskite to yield $\mathrm{Pb}^{0}$ under similar conditions had already been proposed..$^{22}$ Our observations indicate that the transformation that MAPBr undergoes when subjected to ultrahigh vacuum and light of energy, $h \nu$, higher than the electronic bandgap, $E_{g}$, is consistent with the following chemical equation

$$
\mathrm{CH}_{3} \mathrm{NH}_{3} \mathrm{PbBr}_{3} \stackrel{h \nu>E_{\mathrm{g}}}{\longrightarrow} \mathrm{Pb}^{0}+\mathrm{CH}_{3} \mathrm{NH}_{3} \mathrm{Br} \uparrow+\mathrm{Br}_{2} \uparrow
$$

where the following photochemical processes are taking place

$$
\begin{aligned}
& 2 \mathrm{Br}^{-}+2 \mathrm{~h}^{+} \rightarrow 2 \mathrm{Br}^{\bullet} \rightarrow \mathrm{Br}_{2} \uparrow \\
& \mathrm{Pb}^{2+}+2 \mathrm{e}^{-} \rightarrow \mathrm{Pb}^{0}
\end{aligned}
$$

This implies degradation of the lattice, in good agreement with the gradual PL intensity decay versus time observed in the absence of oxygen, as shown in Figure 1a (black solid line). It is at the same time compatible with the increase of halogen vacancies reported in ref 18 .

However, as soon as oxygen is introduced in the chamber (phase I), the $\mathrm{Pb}^{0}$ signal almost disappears and only $\mathrm{Pb}^{2+}$ is detected (see Figure S3). Simultaneously, signals corresponding to oxygen species become very strong, evidencing the adsorption of these species on the perovskite surface. The evolution of these signals is monitored through the binding energy dispersion of the photoelectrons extracted from the $1 \mathrm{~s}$ orbital of oxygen ( $\mathrm{O} 1 \mathrm{~s})$. A selection of the photoelectron energy spectra, taken after different photoexcitation and $\mathrm{O}_{2}$ exposure times, is displayed in Figure 3a. In order to fit these $\mathrm{O}$ 1s spectra, three different components must necessarily be considered, which are assigned to oxide $\left(\mathrm{O}^{2-}\right)$ at $530.0 \pm 0.1$ $\mathrm{eV}$, water $\left(\mathrm{H}_{2} \mathrm{O}\right)$ at $533.0 \pm 0.1 \mathrm{eV}$, and superoxide $\left(\mathrm{O}_{2}^{-}\right)$at $531.8 \pm 0.1 \mathrm{eV}$. Symmetric Gaussian and Lorentzian product functions have been used as line profiles. Peak positions and widths have been kept constant for all fittings, and their full physical meaning has been carefully confirmed. In this regard,
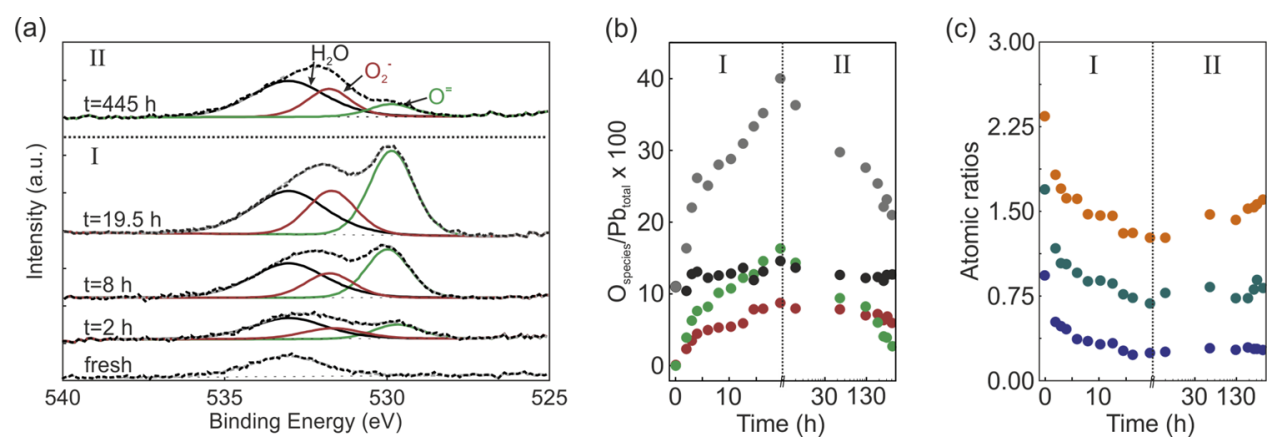

Figure 3. (a) Deconvolution of the $\mathrm{O} 1 \mathrm{~s}$ signals acquired from the photoexcited sample (phase $\mathrm{I}$ ) or from the same sample after keeping it in the dark at ultrahigh vacuum after the treatment (phase II). Fitting of the spectra is realized assuming different species: $\mathrm{H}_{2} \mathrm{O}$ at $533.0 \pm 0.1 \mathrm{eV}, \mathrm{O}_{2}^{-}$at $531.8 \pm 0.1 \mathrm{eV}$, and $\mathrm{O}^{2-}$ at $530.0 \pm 0.1 \mathrm{eV}$. (b) Evolution of the $\mathrm{O}_{\text {total }} / \mathrm{Pb}_{\text {total }}$ (gray circles), $\mathrm{H}_{2} \mathrm{O} / \mathrm{Pb}_{\text {total }}$ (black circles), $\mathrm{O}_{2}{ }^{-} / \mathrm{Pb}_{\text {total }}($ red circles), and $\mathrm{O}^{2-} / \mathrm{Pb}_{\text {total }}$ (green circles) atomic ratios for the whole experiment. (c) Evolution of the $\mathrm{Br} / \mathrm{Pb}$ (orange circles), $\mathrm{C} / \mathrm{Pb}$ (dark green circles), and $\mathrm{N} / \mathrm{Pb}$ (blue circles) atomic ratios for the whole experiment. Please note the horizontal (time) axis is represented in logarithmic scale for panels (b) and (c). 
please notice that the $\mathrm{O}$ 1s electrons in hydroxide $\left(\mathrm{OH}^{-}\right)$and peroxide $\left(\mathrm{O}_{2}^{2-}\right)$ present a similar binding energy as that in superoxide species. However, the presence of $\mathrm{OH}^{-}$and $\mathrm{O}_{2}^{2-}$ is unlikely due to the much higher energies required for their formation; ${ }^{23,24}$ therefore, we can consistently assume that the main contribution to this $\mathrm{O} 1 \mathrm{~s}$ signal comes from the $\mathrm{O}_{2}^{-}$ species. $\mathrm{H}_{2} \mathrm{O}, \mathrm{O}^{2-}$, and $\mathrm{O}_{2}^{-}$signals increase significantly as the light and oxygen soaking treatment evolves, as can be seen in the panels labeled phase I in Figure 3a. The variations of each one of the oxygen species with respect to lead, i.e., $\mathrm{H}_{2} \mathrm{O} / \mathrm{Pb}$ (black circles), $\mathrm{O}^{2-} / \mathrm{Pb}$ (green circles), and $\mathrm{O}_{2}^{-} / \mathrm{Pb}$ (red circles), are shown in Figure $3 \mathrm{~b}$. Oxides and superoxides are the ones that increase more rapidly, while water content grows gradually. Concurrently, the amounts of bromine, carbon, and nitrogen significantly decrease during phase $\mathrm{I}$, as can be seen in Figure $3 \mathrm{c}$, in which we plot the evolution of the $\mathrm{Br} / \mathrm{Pb}$ (orange circles), $\mathrm{C} / \mathrm{Pb}$ (dark green circles), and $\mathrm{N} / \mathrm{Pb}$ (blue circles). In fact, after $20 \mathrm{~h}$ of combined photoexcitation and oxygen exposure, the nitrogen signal has almost disappeared and the carbon one has dropped significantly. These observations are consistent with the following processes occurring at the surface

$$
\begin{aligned}
& \mathrm{CH}_{3} \mathrm{NH}_{3} \mathrm{PbBr}_{3} \underset{\mathrm{O}_{2}}{\stackrel{h \nu>E_{\mathrm{g}}}{\rightarrow}} \\
& {\left[\begin{array}{l}
\mathrm{O}_{2}+\mathrm{e}^{-} \rightarrow \mathrm{O}_{2}^{-} \\
\mathrm{O}_{2}^{-}+\mathrm{e}^{-} \rightarrow \mathrm{O}_{2}^{2-} \\
\mathrm{Pb}^{2+}+\mathrm{O}_{2}^{2-} \rightarrow \mathrm{PbO}+1 / 2 \mathrm{O}_{2} \\
2 \mathrm{Br}^{-}+2 \mathrm{~h}^{+} \rightarrow 2 \mathrm{Br}^{\bullet} \rightarrow \mathrm{Br}_{2} \uparrow \\
2 \mathrm{CH}_{3} \mathrm{NH}_{3}^{+}+\mathrm{O}_{2}^{2-} \rightarrow 2 \mathrm{CH}_{3} \mathrm{NH}_{2} \uparrow+\mathrm{H}_{2} \mathrm{O}+1 / 2 \mathrm{O}_{2}
\end{array}\right.}
\end{aligned}
$$

The formation of adsorbed $\mathrm{O}_{2}^{-}$upon illumination of the perovskite is a central concept to understand the origin of the ion migration effects reported and their relation to the transient PL observed. The adsorption of $\mathrm{O}_{2}$ and its subsequent transformation in $\mathrm{O}_{2}^{-}$implies that oxygen molecules have a greater electron affinity than the perovskite semiconductor work function, and it is strongly favored if the sample is photoexcited as the amount of electrons available increases. In fact, photostimulated oxygen adsorption on the surface of semiconductors is a well-known phenomenon, profusely described and discussed. ${ }^{25}$

In this context, the presence of adsorbed superoxide species and others that can derive from them, such as peroxides $\left(\mathrm{O}_{2}^{2-}\right)$ and hydroxides $\left(\mathrm{OH}^{-}\right)$, can provide the driving force for the photoinduced ion migration phenomena reported in the presence of oxygen, as well as open a path for degradation of the lattice. The proposed mechanism would be as follows:

(i) At short times $\left(t \ll \frac{1}{k_{\mathrm{a}}}\right)$ after photoexcitation starts, $\mathrm{O}_{2}^{-}$ species form on the semiconductor surface as a result of the electron capture by $\mathrm{O}_{2}$ molecules, while interstitial halogen is formed close by hole capture by the halide anions in the perovskite lattice. ${ }^{18}$ In the specific case of $\mathrm{MAPBr}$, the depletion of the bromine, carbon, and nitrogen XPS signals also indicates that volatile molecular bromine and methylamine may be produced, revealing that degradation of the lattices starts as soon as the sample is exposed to oxygen and light, even if it is not noticeable in the evolution of the PL.

(ii) After some time $\left(t \approx \frac{1}{k_{\mathrm{a}}}\right)$, the surface density of $\mathrm{O}_{2}^{-}$is high enough as to activate, by electrostatic repulsion, the migration of interstitial halide anions away from the surface and toward the bulk, ${ }^{26}$ thus favoring the annihilation of halide vacancy/interstitial (in our case, $\mathrm{V}_{\mathrm{I}}^{+} / \mathrm{Br}_{\mathrm{I}}$ ) Frenkel pairs, identified by De Angelis et al. to be relatively abundant in metal halide perovskites. ${ }^{27}$ This temporary reduction of the density of nonradiative traps in the bulk of the material is the reason for the reinforcement of the PL observed.

(iii) For longer times $\left(t \gg \frac{1}{k_{\mathrm{a}}}\right)$, the degradation caused by reaction of a fraction of the $\mathrm{O}_{2}^{-}$anions with the perovskite, via formation of $\mathrm{O}_{2}^{2-}$, overcomes the healing effect caused by defect annihilation associated with ion migration to the bulk of the semiconductor and eventually destroys the perovskite, causing the partially irreversible decrease of the PL reported.

These conclusions are further supported by XPS analysis of the samples after the simultaneous exposure to light and oxygen has ended (phase II). While resting in the dark inside of the XPS apparatus, the evolution of their surface is again periodically analyzed. Interestingly, all three $\mathrm{O} 1 \mathrm{~s}$ signals, although weaker, are still present for times $>100$ s of hours (panel labeled II in Figure 3a), revealing the stability of the photogenerated oxygen species. The concentration of $\mathrm{O}^{2-}$ is the one that drops more rapidly with time, while both $\mathrm{H}_{2} \mathrm{O}$ and $\mathrm{O}_{2}^{-}$are more stable and remain. Signals corresponding to $\mathrm{C}$ and $\mathrm{N}$ are not recovered (dark green and blue circles, respectively, in Figure 3c, phase II), in good agreement with the formation of volatile methylamine. However, in accordance with our proposed mechanism of halide ion migration, part of the bromine returns to the surface, as the recovery of the $\mathrm{Br} 3 \mathrm{~d}$ peak indicates (orange circles in Figure 3c).

The mechanism of ion migration and temporary lattice restoration based on the formation of a surface layer of negatively charged oxygen species herein proposed consistently explains the main phenomena observed when photoexcited organic metal halide films are exposed to oxygen. Also, it integrates the hypothesis proposed by others to explain the different aspects involved. Photodegradation of MAPI was attributed to the reaction of superoxide $\left(\mathrm{O}_{2}^{-}\right)$species, first observed by Haque et al. ${ }^{28}$ However, neither the PL enhancement initially observed, before degradation begins, nor the concomitant illumination-induced halide ion migration phenomena reported were addressed. Also, a variety of phenomena related to light-induced PL enhancement had been hypothesized, such as the partial restoration of the lattice by light-induced annihilation of halide Frenkel pairs, ${ }^{27}$ for which our model provides a driving force. In fact, the presence of a negatively charged surface layer that would give rise to ion redistribution that can lead to PL enhancement had been speculated by Stranks et al. ${ }^{16}$ Our model actually identifies these charged surface species and the precise mechanism by which they are formed in the presence of oxygen if photoexcitation occurs. Please notice that this picture is fully compatible with previous reports on fast molecular oxygen diffusion through the intergranular space in perovskite films and later conversion to superoxide on the semiconductor domain surface, as hypothesized in ref 15 , which has been shown to take place on the order of minutes. The possibility that adsorbed molecular oxygen may act as an electron scavenger to form superoxide species capable of passivating surface iodide vacancies has recently been demonstrated on the grounds of density functional theory calculations, ${ }^{29}$ in full 
agreement with our observations. Finally, it has been recently proposed $^{30}$ that upon light irradiation certain iodide-based perovskites, MAPI and $\mathrm{FA}_{0.7} \mathrm{MA}_{0.25} \mathrm{Cs}_{0.05} \mathrm{PbI}_{3}$, undergo a lattice expansion that is responsible for red-shifted and improved PL as well as better photovoltaic performance. Further, for the mixed-cation perovskite, no evidence for ion migration was found. While such lattice modification may be present in $\mathrm{MAPBr}$, its magnitude is expected to be much smaller as we did not find any spectral changes upon light irradiation (see Figure S6 in the Supporting Information).

In conclusion, we have demonstrated that the presence of oxygen in the environment plays a crucial role in the PL activation of organic metal halide perovskites, the emitted intensity being practically null under truly inert environments, i.e., under ultrahigh vacuum. By combining in situ PL and XPS analysis, we have studied the chemical modifications that take place on the surface of organic metal halide perovskites when they are photoexcited in the presence of oxygen. On the basis of these analyses and previous well-established facts, we can rationalize the observed evolution of the photoemission properties of organic metal halide perovskites with time at different fixed oxygen atmospheres. We find experimental evidence that supports the creation of a negatively charged layer of adsorbed anionic oxygen species on the surface of the organic metal halide perovskite and propose a mechanism that provides a driving force for the observed migration of halide anions away from the illuminated areas toward the bulk of the material, where the PL is enhanced. It also explains the gradual and parallel degradation of the lattice that, eventually, has a deleterious effect on the PL. We believe our findings can help understand better the fundamental optically induced chemical processes that occur in these semiconductors and hence to solve the instability associated with them, which is a task of great relevance in order to fulfill the high technological expectations that they generate.

\section{EXPERIMENTAL METHODS}

Preparation of Perovskite Films. Films were deposited on silicon or glass cover slides. Both types of substrates were treated with $\mathrm{O}_{2}$ plasma cleaner. MAPBr films were prepared following the nanocrystal pinning technique described in ref 31 . A $40 \%$ (w/ $\mathrm{w}$, total) solution of $\mathrm{CH}_{3} \mathrm{NH}_{3} \mathrm{Br}$ and $\mathrm{PbBr}_{2}$ (1:1 molar ratio) in dimethyl sulfoxide was prepared by dissolving appropriate amounts of the compounds. The precursor solution was deposited by spin coating (Ossila Limited) following a twostep procedure: $7 \mathrm{~s}$ at $500 \mathrm{rpm}$ and $90 \mathrm{~s}$ at $3000 \mathrm{rpm}$. During the second stage, chloroform was added drop by drop to the spinning substrate. Finally, samples were treated at $90{ }^{\circ} \mathrm{C}$ for $10 \mathrm{~min}$.

Optical Characterization. The temporal evolution of the maximum of the PL spectrum from a MAPBr film shown in Figure 1a was measured using a homemade setup in which a continuous wave (CW) diode laser $(\lambda=450 \mathrm{~nm}, 6.9 \mathrm{~mW})$ was used to excite the sample through an achromatic lens $(f=30$ $\mathrm{cm}$ ), which served also as collection optics to record the PL through a fiber-coupled spectrophotometer (Ocean Optics USB2000). The optical setup was located outside of the XPS chamber, and access was granted through a transparent quartz window.

Fitting of the PL Transients. The activation constant of the PL was extracted by fitting the PL dynamics from the initial background level $\left(\mathrm{PL}_{0}\right)$ to half of the maximum $\mathrm{PL}$ value achieved upon illumination $\left(\mathrm{PL}_{\max }\right)$ using an exponential growth model.

XPS Experiments. The surface of the MAPBr thin films was explored by XPS with an ESCALAB 1 (Vacuum Generators) instrument, which was upgraded with a SPECS Phoibos 100 DLD hemispherical electron energy analyzer. It consisted of a main ultrahigh vacuum chamber connected to two preparation ones. The base vacuum of the main chamber was $<10^{-9} \mathrm{mbar}$, while the base vacuum of the preparation ones was $<10^{-8}$ mbar. To prevent their surface from contamination/corrosion by atmospheric reagents, $\left(\mathrm{H}_{2} \mathrm{O}, \mathrm{O}_{2}, \mathrm{CO}_{2}\right.$, hydrocarbons, etc.), samples were glued onto the sample holder inside of the glovebox within a few minutes after their synthesis and immediately transferred to one of the preparation chambers of the XPS spectrometer inside of doubled-sealed plastic bags filled with $\mathrm{N}_{2}$. The X-radiation used to excite the samples was $\operatorname{Mg~K} \alpha(h \nu=1253.6 \mathrm{eV})$.

Atomic ratios of the detected elements at the surface of the samples were determined from the intensity of their main photoemission peaks: $\mathrm{O} 1 \mathrm{~s}, \mathrm{~N}$ 1s, $\mathrm{C} 1 \mathrm{~s}, \mathrm{Br} 3 \mathrm{~d}$, and $\mathrm{Pb} 4 \mathrm{f}$. For all of these photoemission signals, lineal backgrounds were used to determine the area under the peaks, and later on, these raw intensities were corrected for their calculated atomic sensitivity factors, which take into consideration the inelastic mean free path of each particular photoelectron, ${ }^{32}$ the photoionization cross sections of each atomic orbital, ${ }^{33}$ and the transmission function of the electron kinetic energy analyzer. ${ }^{34}$ It must be noted that all three parameters are currently far from being error-free, ${ }^{35}$ and large divergences between calculated and empirical sensitivity factors have been reported. Consequently, the absolute atomic percentages derived from them are only a first approximation to the real ones, and they do not allow one to get a chemical formula for the composition of the surface. However, they are good enough to follow the evolution of the atomic ratios, with relative errors lower than $\pm 1 \%$.

\section{ASSOCIATED CONTENT}

\section{S Supporting Information}

The Supporting Information is available free of charge on the ACS Publications website at DOI: 10.1021/acs.jpclett.8b01830.

Additional XPS results and PL spectra of the MAPBr film before and after photoactivation in the presence of oxygen (PDF)

\section{AUTHOR INFORMATION}

\section{Corresponding Author}

*E-mail: h.miguez@csic.es.

ORCID ${ }^{\circ}$

Miguel Anaya: 0000-0002-0384-5338

Mauricio E. Calvo: 0000-0002-1721-7260

Juan P. Espinós: 0000-0002-3053-0841

Hernán Míguez: 0000-0003-2925-6360

\section{Author Contributions}

${ }^{\S}$ M.A. and J.F.G.-L. contributed equally.

\section{Notes}

The authors declare no competing financial interest. 


\section{ACKNOWLEDGMENTS}

Financial support of the Spanish Ministry of Economy and Competitiveness under Grant MAT2017-88584-R is gratefully acknowledged. M.A. is grateful to "La Caixa" Foundation for its financial support.

\section{REFERENCES}

(1) Kojima, A.; Teshima, K.; Shirai, Y.; Miyasaka, T. Organometal Halide Perovskites as Visible-Light Sensitizers for Photovoltaic Cells. J. Am. Chem. Soc. 2009, 131, 6050-6051.

(2) Im, J. H.; Lee, C. R.; Lee, J. W. Q.; Park, S. W.; Park, N. G. Efficient Perovskite Quantum-Dot-Sensitized Solar Cell. Nanoscale 2011, 3, 4088-4093.

(3) Lee, M. M.; Teuscher, J.; Miyasaka, T.; Murakami, T. N.; Snaith, H. J. Efficient Hybrid Solar Cells Based on Meso-Superstructured Organ-ometal Halide Perovskites. Science 2012, 338, 643-647.

(4) Etgar, L.; Gao, P.; Xue, Z.; Peng, Q.; Chandiran, A. K.; Liu, B.; Nazeeruddin, M. K.; Grätzel, M. Mesoscopic $\mathrm{CH}_{3} \mathrm{NH}_{3} \mathrm{PbI}_{3} / \mathrm{TiO}_{2}$ Heterojunction Solar Cells. J. Am. Chem. Soc. 2012, 134, 1739617399.

(5) Tan, Z.-K.; Moghaddam, R. S.; Lai, M. L.; Docampo, P.; Higler, R.; Deschler, F.; Price, M.; Sadhanala, A.; Pazos, L. M.; Credgington, D.; et al. Bright Light-Emitting Diodes Based on Organometal Halide Perovskite. Nat. Nanotechnol. 2014, 9, 687-692.

(6) Gonzalez-Pedro, V.; Juarez-Perez, E. J.; Arsyad, W. S.; Barea, E. M.; Fabregat-Santiago, F.; Mora-Sero, I.; Bisquert, J. General Working Principles of $\mathrm{CH}_{3} \mathrm{NH}_{3} \mathrm{PbX}_{3}$ Perovskite Solar Cells. Nano Lett. 2014, 14, 888-893.

(7) Frost, J. M.; Butler, K. T.; Brivio, F.; Hendon, C. H.; van Schilfgaarde, M.; Walsh, A. Atomistic Origins of High-Performance in Hybrid Halide Perovskite Solar Cells. Nano Lett. 2014, 14, 25842590.

(8) Stranks, S. D.; Burlakov, V. M.; Leijtens, T.; Ball, J. M.; Goriely, A.; Snaith, H. J. Recombination Kinetics in Organic-Inorganic Perovskites: Excitons, Free Charge, and Subgap States. Phys. Rev. Appl. 2014, 2, 034007.

(9) Stranks, S. D. Nonradiative Losses in Metal Halide Perovskites. ACS Energy Lett. 2017, 2, 1515-1525.

(10) Leijtens, T.; Eperon, G. E.; Noel, N. K.; Habisreutinger, S. N.; Petrozza, A.; Snaith, H. J. Stability of Metal Halide Perovskite Solar Cells. Adv. Energy Mater. 2015, 5, 1500963.

(11) Galisteo-López, J. F.; Anaya, M.; Calvo, M. E.; Míguez, H. Environmental Effects on the Photophysics of Organic-Inorganic Halide Perovskites. J. Phys. Chem. Lett. 2015, 6, 2200-2205.

(12) Tian, Y.; Peter, M.; Unger, E.; Abdellah, M.; Zheng, K.; Pullerits, T.; Yartsev, A.; Sundström, V.; Scheblykin, I. G. Mechanistic Insights into Perovskite Photoluminescence Enhancement: Light Curing with Oxygen Can Boost Yield Thousand Fold. Phys. Chem. Chem. Phys. 2015, 17, 24978-24987.

(13) Fang, H.-H.; Adjokatse, S.; Wei, H.; Yang, J.; Blake, G. R.; Huang, J.; Even, J.; Loi, M. A. Ultrahigh Sensitivity of Methylammonium Lead Tribromide Perovskite Single Crystals to Environmental Gases. Sci. Adv. 2016, 2, e1600534.

(14) Motti, S. G.; Gandini, M.; Barker, A. J.; Ball, J. M.; Srimath Kandada, A. R.; Petrozza, A. Photoinduced Emissive Trap States in Lead Halide Perovskite Semiconductors. ACS Energy Lett. 2016, 1, $726-730$.

(15) Aristidou, N.; Eames, C.; Sanchez-Molina, I.; Bu, X.; Kosco, J.; Islam, M. S.; Haque, S. A. Fast Oxygen Diffusion and Iodide Defects Mediate Oxygen-Induced Degradation of Perovskite Solar Cells. Nat. Commun. 2017, 8, 15218.

(16) deQuilettes, D. W.; Zhang, W.; Burlakov, V. M.; Graham, D. J.; Leijtens, T.; Osherov, A.; Bulovic, V.; Snaith, H. J.; Ginger, D. S.; Stranks, S. D. Photo-Induced Halide Redistribution in Organicinorganic Perovskite Films. Nat. Commun. 2016, 7, 11683.

(17) Galisteo-López, J. F.; Li, Y.; Míguez, H. Three-Dimensional Optical Tomography and Correlated Elemental Analysis of Hybrid Perovskite Microstructures: an Insight into Defect-Related Lattice
Distortion and Photoinduced Ion Migration. J. Phys. Chem. Lett. 2016, 7, 5227-5234.

(18) Kim, G. Y.; Senocrate, A.; Yang, T.-Y.; Gregori, G.; Grätzel, M.; Maier, J. Large Tunable Photoeffect on Ion Conduction in Halide Perovskites and Implications for Photodecomposition. Nat. Mater. 2018, 17, 445-449.

(19) Meggiolaro, D.; Mosconi, E.; De Angelis, F. Mechanism of Reversible Trap Passivation by Molecular Oxygen in Lead-Halide Perovskites. ACS Energy Lett. 2017, 2, 2794-2798.

(20) Grancini, G.; D’Innocenzo, V.; Dohner, E. R.; Martino, N.; Srimath Kandada, A. R.; Mosconi, E.; De Angelis, F.; Karunadasa, H. I.; Hoke, E. T.; Petrozza, A. $\mathrm{CH}_{3} \mathrm{NH}_{3} \mathrm{PbI}_{3}$ Perovskite Single Crystals: Surface Photophysics and Their Interaction with the Environment. Chem. Sci. 2015, 6, 7305-7310.

(21) Eames, C.; Frost, J. M.; Barnes, P. R. F.; O’Regan, B. C.; Walsh, A.; Islam, M. S. Ionic Transport in Hybrid Lead Iodide Perovskite Solar Cells. Nat. Commun. 2015, 6, 7497.

(22) Tang, X.; Brandl, M.; May, B.; Levchuk, I.; Hou, Y.; Richter, M.; Chen, H.; Chen, S.; Kahmann, S.; Osvet, A.; et al. Photoinduced Degradation of Methylammonium Lead Triiodide Perovskite Semiconductors. J. Mater. Chem. A 2016, 4, 15896-15903.

(23) Ruscic, B.; Wagner, A. F.; Harding, L. B.; Asher, R. L.; Feller, D.; Dixon, D. A.; Peterson, K. A.; Song, Y.; Qian, X.; Ng, C.-Y.; et al. On the Enthalpy of Formation of Hydroxyl Radical and Gas-Phase Bond Dissociation Energies of Water and Hydroxyl. J. Phys. Chem. A 2002, 106, 2727-2747.

(24) Ervin, K. M.; Anusiewicz, I.; Skurski, P.; Simons, J.; Lineberger, W. C. The Only Stable State of $\mathrm{O}_{2}^{-}$Is the $\mathrm{X}^{2} \Pi_{\mathrm{g}}$ Ground State and It (Still!) Has an Adiabatic Electron Detachment Energy of $0.45 \mathrm{eV}$. J. Phys. Chem. A 2003, 107, 8521-8529.

(25) Pankove, J. L. Optical Processes in Semiconductors; Dover Publications Inc.: New York, 1975; pp 302-333 and pp 352-366.

(26) Yuan, Y.; Huang, J. Ion Migration in Organometal Trihalide Perovskite and Its Impact on Photovoltaic Efficiency and Stability. Acc. Chem. Res. 2016, 49, 286-293.

(27) Mosconi, E.; Meggiolaro, D.; Snaith, H. J.; Stranks, S. D.; De Angelis, F. Light-Induced Annihilation of Frenkel Defects in OrganoLead Halide Perovskites. Energy Environ. Sci. 2016, 9, 3180-3187.

(28) Aristidou, N.; Sanchez-Molina, I.; Chotchuangchutchaval, T.; Brown, M.; Martinez, L.; Rath, T.; Haque, S. A. The Role of Oxygen in the Degradation of Methylammonium Lead Trihalide Perovskite Photoactive Layers. Angew. Chem., Int. Ed. 2015, 54, 8208-8212.

(29) Brenes, R.; Eames, C.; Bulović, V.; Islam, M. S.; Stranks, S. D. The Impact of Atmosphere on the Local Luminescence Properties of Metal Halide Perovskite Grains. Adv. Mater. 2018, 30, 1706208.

(30) Tsai, H.; Asadpour, R.; Blancon, J.-C.; Stoumpos, C. C.; Durand, O.; Strzalka, J. W.; Chen, B.; Verduzco, R.; Ajayan, P. M.; Tretiak, S.; et al. Light-induced lattice expansion leads to highefficiency perovskite solar cells. Science 2018, 360, 67-70.

(31) Cho, H.; Jeong, S. H.; Park, M. H.; Kim, Y. H.; Wolf, C.; Lee, C. L.; Heo, J. Y.; Sadhanala, A.; Myoung, N.; Yoo, S.; et al. Overcoming the Electroluminescence Efficiency Limitations of Perovskite Light-Emitting Diode. Science 2015, 350, 1222-1225.

(32) Tanuma, S.; Powell, C. J.; Penn, D. R. Calculations of Electron Inelastic Mean Free Paths. IX. Data for 41 Elemental Solids Over the $50 \mathrm{eV}$ to $30 \mathrm{keV}$ Range. Surf. Interface Anal. 2011, 43, 689-713.

(33) Scofield, J. H. Hartree-Slater Subshell Photoionization Crosssections at 1254 and $1487 \mathrm{eV}$. J. Electron Spectrosc. Relat. Phenom. 1976, 8, 129-137.

(34) Transmission function curves were provided by SPECS, the manufacturer of the hemispherical electron energy analyzer, a Phoibos 100

(35) Seah, M. P. Quantification of AES and XPS. In Practical Surface Analysis by Auger and X-ray Photoelectron Spectroscopy; Briggs, D., Seah, M. P., Eds.; Wiley: Chichester, U.K., 1983. 